\title{
Safety stock placement for serial systems under supply process uncertainty
}

\author{
Bengisu Urlu ${ }^{1}$. Nesim K. Erkip ${ }^{2}$ (D)
}

Published online: 1 January 2020

(c) Springer Science+Business Media, LLC, part of Springer Nature 2020

\begin{abstract}
In this study, we address safety stock positioning when demand per period is a known constant but supply is uncertain. The supply is either available or not available, while the setting is that of a periodically reviewed, serial system following a base stock policy. Each stage is allowed to operate according to the guaranteed or stochastic service model. We use a Discrete Time Markov Chain model for expressing the expected onhand inventories for each stage, along with other terms of interest, as a function of policy parameters determined by a given service level requirement for the end product. Exact models are constructed for single-stage and two-stage systems. As the number of states for a two-stage system grows exponentially, we propose an approximation for expressing the effect of the input stage using a single parameter. A generalization for the approximation is provided for a multi-stage problem. Computational evaluations of the approximation, as well as numerical comparisons of different cases, are presented.
\end{abstract}

Keywords Inventory management $\cdot$ Safety stock positioning $\cdot$ Supply uncertainty $\cdot$ Serial systems

\section{Introduction}

The safety stock placement problem involves determining the right amount of base stock in each stage, to ensure the required service level or the lowest expected cost. A stage may have a stock of finished goods as well as work-in-process inventory to

Bengisu Urlu started conducting this research at Bilkent University. She continued working on it at Eindhoven University of Technology, and finalized at INSEAD.

Bengisu Urlu

bengisu.urlu@insead.edu

Nesim K. Erkip

nesim@bilkent.edu.tr

1 Technology and Operations Management, INSEAD, Fontainebleau, France

2 Department of Industrial Engineering, Bilkent University, Ankara, Turkey 
enable possible production realization. There are many approaches to solve the stock placement problem: Song and Zipkin (2003) and Atan et al. (2017) present comprehensive reviews of undertaken studies, especially on assembly systems; Axsäter (2003) examines divergent systems; Inderfurth and Minner (1998) study service concepts in such systems; and Graves and Willems (2003) investigate more general multi-echelon systems. Among safety stock placement approaches, we use the two regimes presented by Graves and Willems (2003); namely, the stochastic service model (SSM) and the guaranteed service model (GSM). In the SSM, stock placements at each stage are performed according to a function of the stochastic delay from upstream stages. In the GSM, each stage ensures that it can meet its requirement for the next stage by committing to a maximum service time (Graves and Willems 2003). In these models, it is assumed that supply is always available at each stage and at each period of operation.

Supply uncertainty refers to supply disruptions, which may be due to operational inefficiencies as well as intentional or unintentional human actions; hence, it has several forms, as described in Snyder et al. (2016). Yield uncertainty covers the cases where the quantity delivered by a supplier or the amount produced by a manufacturing process is a random variable that depends on the order quantity. Capacity uncertainty, in contrast, covers the cases where the supplier's delivery capacity or the company's manufacturing capacity is a random variable that is generally independent of the order quantity. Transportation time uncertainty occurs due to the stochastic nature of transportation time. Finally, process uncertainty considers the variability of the production processes. In a way, it is similar to yield uncertainty, but one may end up with no production as a result of process variability. There are many studies on delineating supply uncertainty structures. Silver (1976) defines supply uncertainty by allowing the quantity received to be a random variable and a random proportion of the quantity ordered. Shih (1980), Ehrhardt and Taube (1987), and Henig and Gerchak (1990) also regard uncertain supply as a random multiple of the quantity requisitioned. Parlar and Berkin (1991) view supply uncertainty as a situation in which supply is either available or unavailable for a random duration in a continuous review environment. Similarly to our base model, Güllü et al. (1997) define supply uncertainty as supply being either fully available or completely unavailable according to some probabilities, in a periodic review setting. Güllü et al. (1999) extend the same idea and regard supply uncertainty as supply being fully available, partially available, or completely unavailable according to some probabilities. Other pertinent models are described in two review articles by Yano and Lee (1995) and Snyder et al. (2016).

Another related research area is the analysis of tandem queues with finite buffers and unreliable machines. In this area, most of the traditional literature has been devoted to modeling and analysis of such production systems, with the central issues being performance analysis and computability. More recently, studies have considered an objective function that incorporates system performance and the cost of buffers between stages (usually of an automated production system). There is an analogy between this set of problems and the stock positioning problem: Positioning stocks can be considered as holding buffers in tandem queues with finite buffers. The distinction in the literature usually comes in the environmental description of the problem (e.g., type of processes, demand distribution, service distribution, 
etc.), as well as in the objective function. The objective function of a typical serial inventory system considers holding costs which are functions of the stage, whereas in the majority of the tandem queue literature only a constraint on the total buffer usage is considered, assuming equal cost buffers. Nevertheless, there are important overlaps in the analytic approaches, especially when the distributions in question are identical. A recent review of the so-called buffer allocation problem can be found in Weiss et al. (2019). As stated in that review, one area authors claim is "insufficiently captured" by the literature is when there is uncertainty in the supply process, and this is the aspect we aim to study in our current work. There are numerous studies on tandem queues, but here, we refer to a more related and recent set of studies that consider solution algorithms for the buffer space allocation problem (Gershwin and Schor 2000); lines operated with given policies (Wu et al. 2017; Liberopoulos 2018); and, lines with tandem queues and unreliable machines (Lee et al. 2017, 2018).

The main purpose of this research is to consider supply uncertainty for the safety stock placement problem. Our main motivation stems from serial systems, representing an important special case of assembly systems. Serial systems usually operate on a just-in-time basis, meaning that suppliers are expected to deliver in small lots to the next stage and finally to the equipment manufacturer, every day or even more frequently. The equipment manufacturer performs final assembly, which is well-planned, and practically there is no uncertainty in the desired throughput of the assembly line. Moreover, one can claim that the quantity produced per day is a constant, as a result of the assembly line balancing efforts. It is quite common to observe certain suppliers' inability to ship every day, motivating an all-or-nothing type of supply uncertainty. We call this type of uncertainty "process uncertainty", usually resulting from an equipment failure or failure of the second-tier suppliers to supply the necessary input. Note that this structure of process uncertainty is also well studied in tandem queue literature (for examples, see Lee et al. 2017, 2018). What is interesting about the problem is that process uncertainty structure brings in the necessity of safety stock placement in different stages, although demand is not stochastic. A limited number of studies incorporate both supply and demand uncertainty in the same modeling environment. Bollapragada et al. (2004a, b) model the supply process allowing for backlogs but limiting their duration, similar to a restricted GSM approach.

We consider a periodic review system in which each stage has an order-up-to point. Demand per period is $Q$ units, deterministic and known. We assume a supply uncertainty structure where supply is either available or not available, as described by Güllü et al. (1997). The structure, depicted by Bernoulli-type stages, has been considered by the tandem queues literature as well. For example, Diamantidis and Papadopoulos (2004) offer a dynamic programming approach for buffer allocation, and Naebulharam and Zhang (2014), Lee et al. (2017, 2018), address waiting timerestricted products for performance evaluation. Hence, a stage may have sufficient work-in-process to work on, but as a result of process uncertainty, it may not be able to produce. Additionally, if no work-in-process is available, the stage will not be able to produce, following the "starvation" characteristic described mostly by the tandem queue literature. If there is sufficient work-in-process and production is realized, 
then any quantity to bring the stocks to the order-up-to point can be produced. In other words, there is no capacity restriction. Hence, following these characteristics, a stochastic delay term can be defined for items to be available for the next stage. Therefore, the core of the problem is to reflect the stochastic characteristics of the supply uncertainty problem combined with the characteristics of the safety stock placement models.

To fulfill our research purpose, we restrict our analysis to serial systems. Graves and Willems (2005), Hua and Willems (2016a, b) present evidence for the importance of analyzing a two-stage serial system. Gavirneni (2004) describes a chip manufacturer with a four-stage serial system. Although the supply chain of the chip manufacturer is far more complex, the author identify a small number of suppliers responsible for inefficiencies in the operations, and hence it can be reduced to a serial system. Our objective in this research is to obtain exact (and if possible explicit) terms for the considered serial system. We use the two regimes introduced (SSM and GSM) as possible stock placement models, in addition to implementing a supply uncertainty structure in which supply is either fully available or not available at all. Then, we model the system as a Discrete Time Markov Chain (DTMC).

Our main contribution is modeling the stock placement problem in serial systems with supply uncertainty given the presumed structure. Contrary to the available literature, we allow different stages to operate with different service regimes, each stage following either the SSM or the GSM. We compare two-stage systems based on expected safety stock costs, and further compare SSM and GSM regimes. We propose an approximation method as the number of states in the DTMC model grows exponentially.

The paper is organized as follows. Section 2 provides necessary background information. In Sect. 3, we consider single-stage and two-stage problems, operating with the GSM or the SSM. In Sect. 4, we present the approximation scheme and generalization of the approximation to a multi-stage problem. Our computational experiments for the two-stage models and comparisons appear in Sect. 5. Finally, in Sect. 6 we conclude and offer possible generalizations and other extensions for future work.

\section{Background}

\subsection{Supply uncertainty}

In Güllü et al. (1997), an inequality that provides the optimal number of periods $(K)$ to consider during which the cumulative demand is equal to optimal order-up-to level is derived. In our research, we focus on the special case of a similar problem with infinite time horizon. Note that $K$ stands for the number of periods of demand and in our case the order-up-to level is $K Q$. Additionally, the right side of the inequality is represented by a service level definition rather than costs, following the service-level definition made by Silver (1976), and used by Graves and Willems (2003). As a complementary explanation, we can say that each value of $K$ corresponds to a service level. 


\subsection{Approaches to safety stock placement}

Graves and Willems (2003), consider two regimes of the safety stock placement problem, referred as the guaranteed service model (GSM) and the stochastic service model (SSM). They consider the general environment shown in Fig. 1. Note that the aim is to characterize the replenishment times, given that a stage might have multiple unreliable upstream suppliers that might also have unreliable suppliers.

Each regime structure allows for writing an approximate expression of the expected replenishment times. The system operates according to an order-up-to policy where the base stock is given in Graves and Willems (2003). Using these replenishment times, safety stocks can be computed. The total expected safety stock cost can be found and used for performance evaluation for a given set of service level targets (or safety factors) in each stage in an optimization context. Finally, for any regime the purpose is to eventually come up with safety stock terms for each stage to minimize total expected cost of safety stock of the system to satisfy a service level target of the end-product. Note that the safety factors of all stages are decision variables.

Under the SSM regime, Graves and Willems (2003) assume that at most one supplier is allowed to stock out per period, and delay is equal to this supplier's processing time. Thus, they represent replenishment time for a stage as a function of the processing time of the current stage added to the expected value of the processing time from the predecessor stage, causing a stock-out. The probabilities used to compute expectations are not exact and are taken from Ettl et al. (2000). Moreover, demand over the net replenishment time is assumed to be normally distributed with a mean and a standard deviation, that are derived once the expected replenishment time is determined. Note that if we follow the supply uncertainty structure specified in the previous subsection, the implementation of the SSM at Stage $j$ corresponds to finding $K_{j}$, and hence the order-up-to level is $K_{j} Q$.

When it comes to the GSM, the challenge is to determine the maximum service time given a safety factor and a demand upper bound. Service times are

Fig. 1 Representation of multi-

echelon supply chain

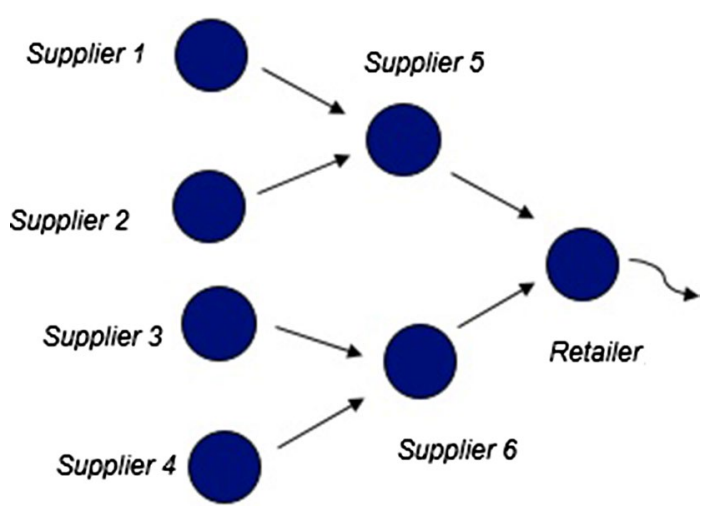


decision variables for internal customers and exogenous input for external customers. Demand is again assumed to be normally distributed and the demand upper bound can be arbitrarily set. In this case, the maximum service times of inbound and outbound stages affect the replenishment time.

In the GSM, if demand exceeds the upper bound, the available safety stock in the system will not be enough to satisfy the service time promise. Graves and Willems (2003) assume that in the case of excess demand, the amount that is above the upper bound can be regarded as loss or outsourced to satisfy customer requirements. However, there is no additional term that represents the outsourced amount in their objective function. An approach to model the case when these assumptions are violated can be found in Rambau and Schade (2014).

Note that if we follow the specified supply uncertainty structure, the implementation of the GSM for any Stage $j$ corresponds to finding order-up-to level $K_{j} Q$, and a maximum service time (in units of periods) promised to satisfy requirements, call it $M_{j}$. Note that $M_{j}>K_{j}$ because the order-up-to level economically should not exceed the maximum number of periods designated for the guaranteed service time. Furthermore, $M_{j}=K_{j}$ means that there will be no backlog.

\subsection{Statement of the problem}

Following the standard literature, we can state our problem as:

Minimize total expected safety stock cost over all stages

subject to: service level constraint for the end product

Given the supply uncertainty structure presumed and supporting two regimes of service (the SSM and the GSM), in the rest of the subsection we detail the above problem statement.

Stages are numbered in a decreasing way, Stage 1 defines the final stage. For each stage we have at most two variables to decide: $K_{j}$ is the order-up-to level, and $M_{j}$ is maximum service time defined in Graves and Willems (2003) (note that if Stage $j$ is operated by the SSM regime, $M_{j}$ becomes infinity).

We depict the order-of-events in Fig. 2 and present the description below. Note that all quantities are integer multiples of $Q$.

- We start any period for Stage $j$ by receiving whatever was sent by Stage $j+1$ lead-time periods ago. Anything received is added to $R M_{j}$ " "raw material" for Stage $j$ (the input needed to produce at Stage $j$ ).

- Stage $j$ observes its finished goods inventory, $F G_{j}$.

- If $F G_{j}>0$,

- demand for Stage $j-1$ is satisfied from stock and arrives at the downstream stage after the appropriate lead time.

- $F G_{j}$ is reduced by one.

- Stage $j$ applies order-up-to production using $K_{j}$. Maximum production is limited by the amount of raw material inventory on hand, $R M_{j}$. 
Produce up to $\boldsymbol{K}_{\boldsymbol{j}}$; production is restricted according to the raw material inventory on hand. If the demand was not satisfied from stock and if production is successful, satisfy the demand after production. If production is not successful, increase the number of periods with delay by one

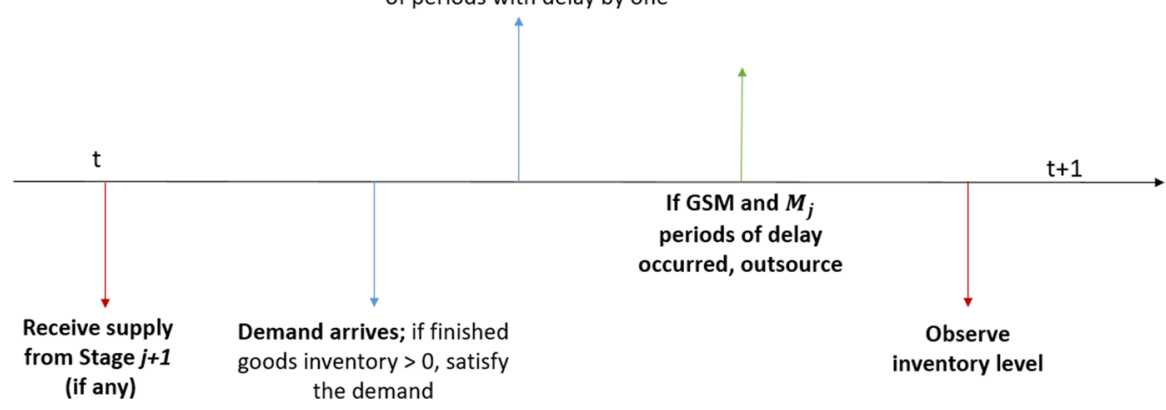

Fig. 2 Order of events for Stage $j$

- If $R M_{j}>0$ and production is successful, update $R M_{j}$ and $F G_{j}$.

- Otherwise, no change.

- If $F G_{j} \leq 0$,

- demand cannot be satisfied from stock.

- $F G_{j}$ is reduced by one.

- Stage $j$ applies order-up-to production. Maximum production is limited by the amount of raw material inventory on hand, $R M_{j}$.

- If $R M_{j}>0$ and production is successful, check $R M_{j}-K_{j}$.

- If $R M_{j}-K_{j}>0, R M_{j}-K_{j}$ units of $F G_{j}$ are sent to Stage $j-1$ to satisfy accumulated backlogs.

- Otherwise if $R M_{j}-K_{j} \leq 0, R M_{j}$ units of $F G_{j}$ are sent to Stage $j-1$, to partially satisfy accumulated backlogs.

- Update $R M_{j}$ and $F G_{j}$.

- If $R M_{j}=0$ or production is not successful: If Stage $j$ operates according to the GSM regime and a delay of $M_{j}$ periods has occurred, then Stage $j$ outsources and sends the unit to Stage $j-1$. To account for the outsourced amount, the finished goods inventory is increased by one and the raw material inventory is decreased by one as it is not needed anymore. If the raw material inventory is zero, the finished goods inventory level of Stage $j+1$ is increased by one (note that the finished goods inventory level of Stage $j+1$ should be negative, and by subcontracting one unit we are eliminating the need for that unit).

The initial stage (the one with the largest index) is assumed to have an uninterrupted infinite supply. Define $h_{j}$ as the holding cost for one finished item in Stage $j$. Define $E\left[I N_{j}\right]$ as the expected ending on-hand inventory for the finished item of Stage $j$ (note that part of the inventory can be located at Stage $j-1$ as raw material waiting for production in Stage $j-1)$. 
Hence we can now rewrite the problem statement:

$$
\min _{K_{j}, M_{j}, \forall j} \sum_{j} h_{j} E\left[I N_{j}\right]
$$

subject to:

Probability of not satisfying the demand at Stage $1 \leq 1$ - service level desired

It is important to point out that expected ending on-hand inventory is safety stock plus expected demand in a period. In the next two sections, we show how we can derive the above expected ending on-hand inventory and the probability terms exactly, given $K_{j}$ and $M_{j}$ for all $j$, for single-stage and serial two-stage systems using DTMC models. Note that these terms are exact, contrary to the available literature. We also derive certain quantities of interest, expected number of backlogs, expected number of subcontracted units (0 if the stage is following the SSM regime), and expected work-in-process inventory (for Stage 1, only for the two-stage system) to be used for computational study. Furthermore, we derive the probability of Stage $j$ satisfying the demand of Stage $j-1$ on time, which we call the probability of supply $\left(\pi^{S_{j}}\right)$. This parameter is instrumental for decomposing the stages, and characterizing service level. Note that Stage 1's (the most downstream stage) probability of supply is equal to the service level faced by the end customer: hence it is the system's service level. Therefore, probability of not satisfying the demand at Stage 1 is the term for the left-hand side of the constraint for the model represented by Eq. (1).

\section{Single-stage and two-stage problems}

\subsection{Single-stage problem}

To correctly reflect the characteristics of the safety stock placement models and the supply uncertainty model, we start with the simplest model, single-stage when demand is stationary and production is uncertain. We assume that the predecessor stage always supplies the needed raw-material, and hence the single-stage considered is operating to satisfy the next stage. We assume that transportation time $(L)$ is deterministic. To simplify the exposition, in the next two subsections it is presumed that transportation time is zero. Following the subsections, we generalize our results for any $L$.

If there is sufficient inventory at the beginning of a period, the next stage's demand is satisfied immediately. However, if there is no inventory and production cannot be realized, there is a delay. Hence, the delivery delay faced by the lower stage is reflected as the replenishment time. Note that having no inventory implies that there have been some consecutive periods of unsuccessful production.

Therefore, the replenishment time is a random variable, $\tau$. Using the all-or-nothing supply, $\tau$ follows a geometric distribution for the SSM regime (or a truncated geometric 
distribution for the GSM), where $p$ is the probability of no production and $i$ period delay is faced with probability $p^{i}(1-p)$.

\subsubsection{Stochastic service model}

The demand during replenishment time also follows a geometric distribution with parameter $p$. The expressions for the distribution of the replenishment time, distribution of demand during replenishment time, expected demand, and standard deviation of demand during replenishment time are presented in Table 1.

To obtain the terms of the model, a DTMC model is used. The states of the Markov Chain are defined as the net inventory levels at the end of a period, following the order of events structure in Fig. 2. Hence, at the beginning of the period, if possible, demand is satisfied. After demand is satisfied, production order is placed up to the order-up-to level. For a given probability of no production, suppose the order-up-to level is given as $K Q$. Hence, when the replenishment time is realized as zero, the ending inventory of period $t$ will be positive, let us say $(K-n) Q$. Inventory level after satisfying the demand will be $(K-n-1) Q$ and ending inventory of period $t$ will again be $K Q$ if the production is successful, since it produces up to the order-up-to level. If not, the state will remain as $(K-n-1) Q$. Replenishment time will take positive values if the inventory level at the end of a period is not positive, and production is not successful for some periods. This setting is a special case of the standard birth and death process. The transition matrix is given in "Appendix 1", when $Q$ is assumed to be 1 to ease the notation.

One can obtain steady-state probabilities using standard analysis where $\pi_{j}$ indicates the long-term probability that there will be $j$ units of inventory at the end of a period. As a result, steady-state probabilities are given by

$$
\pi_{K-n}=p^{n}(1-p) \text { for } n=0, \ldots, \infty
$$

Expected ending on-hand inventory and expected number of backlogs in the system can be written as

$$
E\left[I N_{j}\right]=Q \sum_{i=0}^{K-1}(K-i) \pi_{K-i}=Q\left[K-\frac{p\left(1-p^{K}\right)}{1-p}\right]
$$

Table 1 Details of the SSM for single-stage model

\begin{tabular}{ll}
\hline & SSM \\
\hline Replenishment Time $(\tau)$ & $\tau=\left\{i\right.$ with probability $p^{i}(1-p)$, where $\left.i=0,1,2,3, \ldots\right\}$ \\
Demand during $\tau$ & $D=\left\{i Q\right.$ with probability $p^{i}(1-p)$, where $\left.i=0,1,2,3, \ldots\right\}$ \\
$E\left[D_{\tau}\right]$ & $Q \frac{p}{1-p}$ \\
$\sigma_{\tau}$ & $Q \frac{\sqrt{p}}{1-p}$ \\
\hline
\end{tabular}




$$
E[\text { Backlog }]=p\left(Q \sum_{i=0}^{\infty} i \pi_{-i}\right)=Q \frac{p^{K+2}}{1-p}
$$

\subsubsection{Guaranteed service model}

Similar to the SSM, replenishment time definition for the GSM is characterized by the delay. Delay follows a truncated geometric distribution, where $M$ is the maximum delay to satisfy guaranteed service time.

The demand during replenishment time also follows a truncated geometric distribution with parameter $p$. The expressions for the distribution of the replenishment time, distribution of demand during replenishment time, expected demand and variance of demand during replenishment time are presented in Table 2.

The DTMC model used is similar to the one in the SSM. The only difference is that, if there are $M$ consecutive periods of no production, outsourcing is performed so that the guarantee given can be realized. For the same state definition as in the SSM (net inventory at the end of a period), a probability transition matrix is specified and presented in "Appendix 2". However, in the case of the GSM, the number of states is finite. As an operational decision, outsourcing is done when there are $M$ periods of delay (at state $(-M+K) Q$ ), by satisfying only $Q$ backlogs (i.e. the demand received $M$ periods ago). Then, if production is realized, it produces up to $K Q$, otherwise the state will be $(-M+K) Q$ rather than $(-1-M+K) Q$. Thus, when production is not realized for consecutive $M$ periods, demand is outsourced. Steadystate probabilities are,

$$
\begin{gathered}
\pi_{K-n}=p^{n}(1-p) \text { for } n=0, \ldots, M-1 \\
\pi_{-M+K}=p^{M}
\end{gathered}
$$

Performance measures we are interested-expected ending on-hand inventory, expected number of backlogs and expected number of outsourced units- can be writ-

\begin{tabular}{|c|c|}
\hline & GSM \\
\hline Replenishment Time & $\begin{array}{c}\tau=\left\{\begin{array}{c}i \text { with probability } \quad p^{i}(1-p) \\
M \text { with probability } 1-\sum_{i=0}^{M-1}\left[p^{i}(1-p)\right]\end{array}\right\} \\
\text { where } \mathrm{i}=0,1,2, \ldots, \mathrm{M}-1\end{array}$ \\
\hline Demand during $\tau$ & $\begin{array}{c}D=\left\{\begin{array}{c}i Q \text { with probability } \quad p^{i}(1-p) \\
M Q \text { with probability } 1-\sum_{i=0}^{M-1}\left[p^{i}(1-p)\right]\end{array}\right\} \\
\text { where } \mathrm{i}=0,1,2, \ldots, \mathrm{M}-1\end{array}$ \\
\hline$E\left[D_{\tau}\right]$ & $(1-p) \sum_{i=0}^{M-1} i Q p^{i}+M Q\left(1-\sum_{i=0}^{M-1} p^{i}(1-p)\right)$ \\
\hline$\sigma_{\tau}^{2}$ & $\begin{array}{l}(1-p)\left(\sum_{i=0}^{M-1} i^{2} Q^{2} p^{i}-(1-p)\left(\sum_{i=0}^{M-1} i Q p^{i}\right)^{2}\right)-2 M Q(1-p) \sum_{i=0}^{M-1} i Q p^{i} \\
\left(1-\sum_{i=0}^{M-1} p^{i}(1-p)\right)+M^{2} Q^{2}\left(1-\sum_{i=0}^{M-1} p^{i}(1-p)\right)\left(1-\left(1-\sum_{i=0}^{M-1} p^{i}(1-p)\right)\right)\end{array}$ \\
\hline
\end{tabular}
ten as

Table 2 Details of the GSM for single-stage model 


$$
\begin{gathered}
E\left[I N_{j}\right]=Q \sum_{i=0}^{K-1}(K-i) \pi_{K-i}=Q\left[K-\frac{p\left(1-p^{K}\right)}{1-p}\right] \\
E[\text { Backlog }]=Q p\left(\sum_{i=0}^{M-K-1} i \pi_{-i}+(M-K) \pi_{-M+K}\right) \\
E[\text { Outsourced }]=Q p \pi_{-M+K}=Q p^{M+1}
\end{gathered}
$$

Note that we compute the expected outsourced quantity as given in Eq. (6). We believe this quantity is instrumental for comparing models with the GSM regime to models with the SSM regime.

\subsubsection{Generalization for non-zero lead times}

Now consider $L+\tau$ where $L$ is an integer denoting the transportation time between stages and $\tau$ is the random variable as defined before. $L+\tau$ stands for the replenishment time random variable in the case of non-zero transportation time. Note that when $L$ is different than zero, the order-up-to level will be defined as $(K+L) Q$ for integer $L$ values. Hence, the states will start from $(K+L-L) Q=K Q$, $(K+L-L-1) Q=(K-1) Q$ and so on. Therefore, the transition matrix remains unchanged, and the steady-state probabilities are the same as in the case $L=0$. Then all terms computed for the model remain the same, except that there will be a pipeline inventory to accommodate $L$.

\subsection{Two-stage problem}

When the problem has two echelons, Fig. 3 represents the operations. In this setting, Stage 2 supplies work-in-process inventory to Stage 1, and each stage has its own no production probability as $p_{2}$ and $p_{1}$, given that raw-material inventory is present.

We make two assumptions:

1. It is assumed that there is an ample supply for Stage 2. The service model for Stage 2 can be the SSM or the GSM.

2. Transportation time for a product to reach Stage $1\left(L_{2}\right)$, and transportation time for a product to reach the customer $\left(L_{1}\right)$ are assumed to be zero to ease notation and exposition.

Figure 2 provides the order of events structure for two-stage system. Stage 2 has an order-up-to level $K_{2} Q$, and if GSM, $M_{2}$ denotes the number of consecutive periods with no supply needed to invoke subcontracting. Stage 1 can operate according to the GSM or the SSM. Let $K_{1}$ denote the multiplier of $Q$ for the 


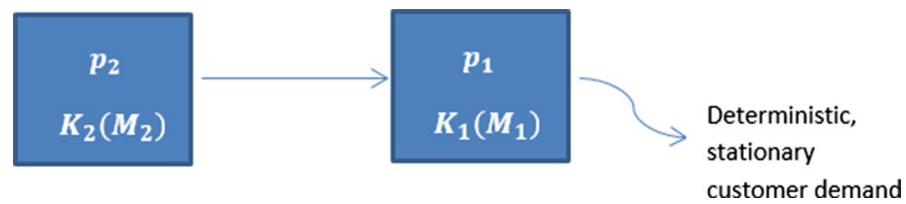

Fig. 3 Two-stage serial system

order-up-to level and $M_{1}$, in the case of the GSM, denote the number of consecutive unsuccessful production attempts needed to invoke subcontracting. Stage 1 can keep work-in-process inventory received from Stage 2, as well as finished goods inventory. Note that in case it has received the supply and cannot produce in a period, Stage 1 carries the work-in-process as inventory (in its inbound).

While modeling the two-stage problem, the impact of Stage 2's operations on Stage 1 should be reflected. To do so, operations of both stages should be tracked and indicated in the transition matrix by characterizing an aggregate state definition for the whole system. $(F G 2, F G 1, R M)$ is defined as the state of DTMC where $F G 2$ represents Stage 2's net finished goods inventory at the end of a period, $F G 1$ represents Stage 1's net finished goods inventory at the end of a period, and $R M$ represents Stage 1's work-in-process inventory (i.e., raw material supplied by Stage 2) on hand at the end of a period (Note that $F G 2$ and $F G 1$ can be positive or negative whereas RM is always non-negative.).

Given our problem structure, we can mention four different regimes for a twostage problem: Let the notation $\mathrm{X} / \mathrm{Y}$ denote the regime followed in a two-stage problem, where $\mathrm{X}$ is the regime for Stage 2 and $\mathrm{Y}$ is the regime for Stage 1; $\mathrm{X}$ and Y can be either SSM or GSM. The analysis below is for a GSM/GSM regime when $Q=1$, for simplicity of exposition.

When both stages operate according to the GSM, possible values of the decision variables are $i=0,1,2, \ldots, M_{2}, j=0,1,2, \ldots, M_{1}$ and $k=0,1,2, \ldots, M_{1}$. $k \leq j$ because raw material accumulates only when Stage 1 cannot produce. If $i>K_{2}$ then, $j \geq i-K_{2}$ as when Stage 2 has negative inventory, it cannot supply to Stage 1. If $i<M_{2}$ then $k+K_{1}-j>0$. If $i=M_{2}$ then $M_{1}>k>K_{1}-j>0$ and other $k$ 's are not possible.

Let the state at the end of period $t$ be $\left(K_{2}-i, K_{1}-j, k\right)$ and at the end of period $t+1$ be $\left(K_{2}-i^{\prime}, K_{1}-j^{\prime}, k^{\prime}\right)$. The following cases can be written:

- Case 1 Both stages can realize production with probability $\left(1-p_{2}\right)\left(1-p_{1}\right)$, then $\left(i^{\prime}, j^{\prime}, k^{\prime}\right)=(0,0,0)$.

- Case 2 Stage 2 can realize production but Stage 1 cannot realize production with probability $\left(1-p_{2}\right) p_{1}$, then Stage 2 supplies all raw material that Stage 1 needs. If $K_{1}-j>0$, Stage 1 needs one unit of raw material. Otherwise, Stage 1 should accumulate enough raw material inventory to raise the finished goods inventory up to $K_{1}$. In this case, Stage 1 needs $K_{1}-F G 1-R M+1$ (FG1 is finished goods inventory at the end of period $t$ at Stage 1, $R M$ is raw material inventory at the end of period $t$ at Stage 1, +1 indicates the demand of Stage 
1 from Stage 2) units. Note that for this example, $F G 1=K_{1}-j$ and $R M=k$. Then the supplied amount is $j-k+1$. Hence,

1. If $K_{1}-j>0,\left(i^{\prime}, j^{\prime}, k^{\prime}\right)=(0, j+1, k+1)$

2. If $j \neq M_{1}$ and $K_{1}-j \leq 0,\left(i^{\prime}, j^{\prime}, k^{\prime}\right)=(0, j+1, j+1)$

3. If $j=M_{1}$ and $K_{1}-j \leq 0,\left(i^{\prime}, j^{\prime}, k^{\prime}\right)=(0, j, j)$

- Case 3 Stage 2 cannot realize production but Stage 1 can realize production with probability $\left(1-p_{1}\right) p_{2}$, then

1. If $i \neq M_{2}$ and $i<K_{2},\left(i^{\prime}, j^{\prime}, k^{\prime}\right)=(i+1, j-k-1,0)$

2. If $i \neq M_{2}$ and $i \geq K_{2},\left(i^{\prime}, j^{\prime}, k^{\prime}\right)=(i+1, j-k+1,0)$

3. If $i=M_{2},\left(i^{\prime}, j^{\prime}, k^{\prime}\right)=\left(M_{2}, j-k, 0\right)$

- Case 4 Both stages cannot realize production with probability $p_{1} p_{2}$, then

1. If $i \neq M_{2}$ and $i<K_{2}, j \neq M_{1} ;\left(i^{\prime}, j^{\prime}, k^{\prime}\right)=(i+1, j+1, k)$

2. If $i \neq M_{2}$ and $i \geq K_{2}, j \neq M_{1} ;\left(i^{\prime}, j^{\prime}, k^{\prime}\right)=(i+1, j+1, k+1)$

3. If $i \neq M_{2}$ and $i<K_{2}, j=M_{1} ;\left(i^{\prime}, j^{\prime}, k^{\prime}\right)=\left(i+1, M_{1}, k\right)$

4. If $i \neq M_{2}$ and $i \geq K_{2}, j=M_{1} ;\left(i^{\prime}, j^{\prime}, k^{\prime}\right)=(i+1, j, k-1)$

5. If $i=M_{2}, j \neq M_{1} ;\left(i^{\prime}, j^{\prime}, k^{\prime}\right)=\left(M_{2}, j+1, k+1\right)$

6. If $i=M_{2}, j=M_{1} ;\left(i^{\prime}, j^{\prime}, k^{\prime}\right)=\left(M_{2}, M_{1}, k\right)$

Following these possible cases, an example transition matrix when $K_{1}=1, M_{1}=2$ and $K_{2}=1, M_{2}=2$ is given in "Appendix 3". Among all possible service model and parameter combinations, this example is the one that has the smallest total number of states ( 8 states). Thus, obtaining the steady-state probabilities is straightforward. In general, for a GSM-GSM system we expect that an exact solution can be computed. However, one can observe that solving problem exactly will suffer from the curse of dimensionality very rapidly as the parameters of the problem get bigger. Furthermore, as the number of states increases and explodes, the accuracy of the computations will be questionable. Note also that for other combinations of service regimes, obtaining an exact solution will either be impossible or require very long computation times.

In the next section, we present an approximation method to obtain the steadystate probabilities.

\section{An approximation to compute steady-state probabilities}

\subsection{Two-stage problem}

For a problem of realistic size, the number of states will explode, making the computation of the exact solution practically impossible. Hence, we propose an approximation. Decomposition and aggregation methods are applied if a huge 
state space makes the numerical solution of Markov Chains too slow or impossible. Weiss et al. (2019) review possible approaches. Decomposition methods are promising as they can be implemented for larger systems. The classical approach of Gershwin (1987) decomposes an $N$-station line into a set of $N-1$ two-station lines. The buffer capacities in the subsystems are the same as in the original line. However, the station characteristics, such as processing times, inter-failure times and repair times are iteratively modified such that the material flow in the subsystem follows the behavior of the original line, and all two-station lines have the same throughput. Hence, conservation of flow is maintained.

Given the special structure of the environment studied, if both stages are operated according to the SSM, we observe the following:

Observation 1 For the SSM, if Stage 2 does not satisfy the "work-in-process" demand of Stage 1 (can be in the form of raw material) in a given period due to lack of inventory, the probability that it will satisfy in the next period is always the same and equal to $\left(1-p_{2}\right)$. Hence, by following the work-in-process inventory and finished goods inventory - a state vector with two elements -, for Stage 1, we can exactly represent the desired structure of the system with the transition probabilities affected by a single term, call it supply probability, $\pi^{S_{j}}$. Note that $\pi^{S_{2}}$ is the probability that Stage 2 is satisfying Stage 1's demand and can simply be computed as the sum of limiting probabilities of those stages that actually supply. Besides, this probability of having a supply at any state is the same for all inventory levels of Stage 1.

Given Observation 1, we are now ready to implement the approximation procedure for an arbitrary two-stage model, where each stage is operated according to either the SSM or the GSM. The method is exact if the supplying stage is operated according to the SSM and approximate for the GSM. Nevertheless, we expect the approximation to be quite accurate.

The second stage of the two-stage environment can be modeled as a DTMC. The state definition for Stage 2 will be the same as for a single-stage model. Let $\pi^{S_{2}}$ be defined as the steady-state probability that there will be a supply from Stage 2. It should be noted that supply is possible when the ending inventory level is above zero or when the stage started with inventory level below (or equal to) zero but production is realized in the period. The first and second terms in Eqs. (7) and (8) indicate these cases, respectively. The third term in the GSM reflects satisfying demand via outsourcing. As a result, using the results of the one-stage problem, $\pi^{S_{2}}$ can be written as

$$
\begin{gathered}
\text { SSM: } \quad \pi^{S_{2}}=\sum_{i=0}^{K_{2}-1} \pi_{\left(K_{2}-i\right)}+\left(1-p_{1}\right) \sum_{i=K_{2}}^{\infty} \pi_{\left(K_{2}-i\right)} \\
\text { and GSM: } \quad \pi^{S_{2}}=\sum_{i=0}^{K_{2}-1} \pi_{\left(K_{2}-i\right)}+\left(1-p_{1}\right) \sum_{i=K_{2}}^{M_{2}-1} \pi_{\left(K_{2}-i\right)}+\pi_{\left(K_{2}-M_{2}\right)}
\end{gathered}
$$

As Stage 1 can keep both finished goods inventory produced and work-in-process inventory received from Stage $2,(i, j)$ is defined as the state of Stage 1 , such that $i$ 
represents the finished goods inventory and $j$ represents the work-in-process inventory at the end of a period. General state transitions can be specified as follows: $t+1$ :

If the state is $\left(K_{1}-i, j\right)$ at the end of period $t$ and $\left(K_{1}-i^{\prime}, j^{\prime}\right)$ at the end of period

Case 1 Production is realized and supply is received with probability $\pi^{S_{2}}\left(1-p_{1}\right)$, then $\left(i^{\prime}, j^{\prime}\right)=(0,0)$.

Case 2 Production is realized but supply is not received with probability $\left(1-\pi^{S_{2}}\right)\left(1-p_{1}\right)$ when $j>0$ then $\left(i^{\prime}, j^{\prime}\right)=(i-j+1,0)$.

Case 3 Production is not realized but supply is received with probability $\pi^{S_{2}} p_{1}$ then $\left(i^{\prime}, j^{\prime}\right)=(i+1, i+1)$.

Case 4 Production is not realized and supply is not received with probability $\left(1-\pi^{S_{2}}\right)$ when $j=0$ then $\left(i^{\prime}, j^{\prime}\right)=(i+1,0)$.

Case 5 Production is not realized and supply is not received with probability $\left(1-\pi^{S_{2}}\right) p_{1}$ when $j>0$ then $\left(i^{\prime}, j^{\prime}\right)=(i+1, j)$.

As stated in the beginning, there is no capacity restriction, and hence when a stage is successful in production, it consumes all the work-in-process inventory available and tries to produce up to $K_{1} Q$ units, which is the order-up-to level. Hence, to reflect this in transition probabilities, the state definition includes the backlogged quantity as well. This can be observed in the transition matrix, given in "Appendix 5" and "7". Let us now consider the two possibilities of operation in Stage 1:

Stage 1 operates according to the SSM. We follow the order of events as given in Fig. 2. Stage 1 operates according to the SSM and Stage 2 can be either the SSM or the GSM, simply represented by the appropriate $\pi^{S_{2}}$. The expressions for the distribution of the replenishment time, distribution of demand during the replenishment time, expected demand, and variance of demand during the replenishment time are presented in "Appendix 4" for the transition matrix in "Appendix 5".

We follow the same sequence to derive equations. Define $\pi_{(i, j)}$ as the steady-state probability of being in state $(i, j)$, indicating the probability of ending a period with $i$ units of net finished goods inventory and $j$ units of work-in-process inventory. One can observe that since the number of states is infinite, some of the steady-state values include infinite sums. However, we observed that all the terms can be written as a function of $\pi_{\left(K_{1}-1,0\right)}$, and approximating infinite sums using truncations, values can be computed numerically. This truncated state is named as a dummy state and when the steady-state values are found, the dummy state is discarded so that remaining steady-state values can be normalized.

Expected ending on-hand inventory is then computed as

$$
E\left[I N_{j}\right]=Q \sum_{i=0}^{K_{1}-1} \sum_{j=0}^{i}\left(K_{1}-i\right) \pi_{\left(K_{1}-i, j\right)}
$$

Expected work-in-process (WIP) inventory on-hand is derived as

$$
E[W I P]=Q \sum_{i=0}^{\infty} \sum_{j=0}^{i} j \pi_{\left(K_{1}-i, j\right)}
$$

Expected backlog can be found by 


$$
\begin{aligned}
E[\text { Backlog }]= & Q\left[\left(1-p_{1}\right) \sum_{i=K_{1}+1}^{\infty} \sum_{j=0}^{i-K_{1}}\left(i-K_{1}+1-j\right) \pi_{\left(K_{1}-i, j\right)}\right. \\
& \left.+p_{1} \sum_{i=K_{1}}^{\infty} \sum_{j=0}^{i}\left(i-K_{1}+1\right) \pi_{\left(K_{1}-i, j\right)}\right]
\end{aligned}
$$

Last, the supply probability of Stage $1\left(\pi^{S_{1}}\right)$ can be defined by using the results of the two-stage problem as,

$$
\pi^{S_{1}}=\sum_{i=0}^{K_{1}-1} \sum_{j=0}^{i} \pi_{\left(K_{1}-i, j\right)}+\left(1-p_{1}\right) \sum_{i=K_{1}}^{\infty} \sum_{j=0}^{i} \pi_{\left(K_{1}-i, j\right)}
$$

Stage 1 operates according to the GSM. Before we proceed, we first need to set a detailed outsourcing policy for Stage 1 that considers the WIP issue, similar to the exact model described in Sect. 3.2. Once production has not been possible for $M_{1}$ consecutive periods and thus finished goods inventory is zero, $Q$ units of finished goods inventory are subcontracted. In this way, only the order that should have been sent $M_{1}$ periods ago is satisfied. Hence, rather than subcontracting the accumulated backlog during the $M_{1}$ periods' delay, we just satisfy the demand for which the maximum service delay $\left(M_{1}\right)$ has occurred. Then, after outsourcing and satisfying the demand, if Stage 1 can perform production and the supply is available, it produces up to $K_{1} Q$. If it cannot realize production, either due to its process uncertainty or because supply is not available, its finished goods inventory stays the same in the transition matrix, and one period demand is outsourced. If production is not realized although supply is available, the work-in-process inventory level becomes $M_{1} Q$ rather than $\left(M_{1}+1\right) Q$ to avoid keeping unnecessary inventory. This can be regarded as Stage 1 giving the extra work-in-process inventory back to the supplier, with zero transition cost, and the outsource facility can be regarded as using this work-in-process inventory to produce one unit of finished good. The expressions for the distribution of the replenishment time, distribution of demand during the replenishment time, expected demand and variance of demand during replenishment time are presented in "Appendix 6".

As an illustration, the transition matrix for Stage 1, constructed using these assumptions for the GSM, can be found in "Appendix 7" ( $Q$ is assumed to be equal to 1, without loss of generality). Steady-state probabilities can be found using this transition matrix, where $\pi_{(i, j)}$ is the probability of being in state $(i, j)$.

Performance measures that interest us (expected ending on-hand inventory, expected WIP inventory on-hand, expected number of backlogs and expected number of outsourced units for Stage 1) are presented below:

$$
E\left[I N_{j}\right]=Q \sum_{i=0}^{K_{1}-1} \sum_{j=0}^{i}\left(K_{1}-i\right) \pi_{\left(K_{1}-i, j\right)}
$$




$$
\begin{gathered}
E[\text { WIP }]=Q \sum_{i=0}^{M_{1}} \sum_{j=0}^{i} j \pi_{\left(K_{1}-i, j\right)} \\
E[\text { Outsourced }]=Q p_{1} \sum_{j=0}^{M_{1}} \pi_{\left(K_{1}-M_{1}, j\right)} \\
E[\text { Backlog }]=Q\left[\left(1-p_{1}\right) \sum_{i=K_{1}+1}^{M_{1}-1} \sum_{j=0}^{i-K_{1}}\left(i-K_{1}+1-j\right) \pi_{\left(K_{1}-i, j\right)}\right. \\
+p_{1} \sum_{i=K_{1}}^{i} \sum_{j=0}^{i}\left(i-K_{1}+1\right) \pi_{\left(K_{1}-i, j\right)} \\
\left.+p_{1}\left(M_{1}-K_{1}\right) \sum_{j=0}^{M_{1}} \pi_{\left(-M_{1}+K_{1}, j\right)}\right]
\end{gathered}
$$

Finally, the supply probability of Stage $1\left(\pi^{S_{1}}\right)$ can be defined by using the results of the two-stage problem as

$$
\pi^{S_{1}}=\sum_{i=0}^{K_{1}-1} \sum_{j=0}^{i} \pi_{\left(K_{1}-i, j\right)}+\sum_{j=0}^{M_{1}} \pi_{\left(K_{1}-M_{1}, j\right)}+\left(1-p_{1}\right) \sum_{i=K_{1}}^{M_{1}-1} \sum_{j=0}^{i} \pi_{\left(K_{1}-i, j\right)}
$$

\subsection{Generalization of the approximation to $\mathrm{N}$-stage problem}

Note that we decomposed the two-stage problem by defining the probability of supply [see Eqs. (7), (8), (12) and (17)]. One should first use Eqs. (7) or (8) for the most upstream stage to obtain the supply probability according to the single-stage model. Then for the next downstream echelon, Eqs. (12) or (17) from the two-stage problem should be used. Once we characterize each stage's supply probability as $\pi^{S_{j}}$, the problem can be sequentially solved for each stage. The number of stages in the model can be more than two by using the upstream stage's supply probability which will correspond to its service level and the downstream stage's state transition probabilities which will be shaped according to supply and process probabilities. The states of the downstream stages will be similar to Stage 1 in our model, possibly with a different $K$ value. Hence, the problem can be solved separately for all stages in a serial network. Once the upstream stage's service level is set, all the other factors will be shaped accordingly. Note that this decomposition enables one to utilize different service models in different stages. A different decomposition method can be found in de Kok and Visschers (1999).

For the computation of the steady-state probabilities of serial $\mathrm{N}$-stage systems under the decomposition scheme proposed, the algorithm below is implemented. Note that each stage can be operated by either the SSM or the GSM. 
Step 1 Solve the $N$ 'th stage by using the single-stage problem to compute steadystate probabilities.

Step 2 Compute $\pi^{S_{N}}$ and call it the upstream stage's supply probability by using Eqs. (7) or (8).

Step 3 Go to the next downstream stage. By using the upstream stage's supply probability, solve the two-echelon problem and obtain steady-state values.

Step 4 Compute $\pi^{S_{N-1}}$ and call it the upstream stage's supply probability by using Eqs. (12) or (17).

Step 5 Repeat step 3 by using the supply probability of the upstream stage, for the computations of the next downstream stage until all stages are covered.

\section{Numerical experiments and evaluation of results}

In the next subsection, we numerically investigate the quality of the approximation proposed in Sect. 4. In Sect. 5.2, we present the results of a numerical experiment we conducted, in which we enumerate all decision variables and compute performance measures.

\subsection{Quality of approximation}

The main motivation of the proposed approximation is to avoid the curse of dimensionality. To test the quality of the approximation, we make the following observations.

Observation 2 As $K$ increases the probability of supply is going to increase, and hence the relative effect of an error in computing will have a tendency to decrease.

Observation 3 As $M$ for the GSM increases, the system looks more like the SSM. The approximation proposed is exact when the SSM is used; hence, we expect the relative error in the approximation to have a decreasing tendency as $M$ increases.

Observation 4 Following Observation 2 and Observation 3, a two-stage system where both stages are operated according to the GSM, with smallest possible $K$ and $M$ values, is expected to yield the largest error for the proposed approximation. Hence, we set $K_{1}=K_{2}=1$ and $M_{1}=M_{2}=2$ for our numerical experiments.

Note that we analyze the exact solution described in Sect. 3.2 and the approximation described in Sect. 4.1. Thus, we compare the exact solution for Stage 1 with the approximation computed by the algorithm given in Sect. 4.2.

Table 3 presents the results for various $p_{1}, p_{2}$ combinations. As can be observed, the approximation performs quite well. When the probability of no production increases, the gap between the results of the exact solution and the approximation increases. However, even in that case the approximation is accurate up to two digits. More important, quantities that are likely to be used as parts of any performance measure, which are listed in the last four columns of Table 3, indicate that the approximation seems to be almost perfect. 
Table 3 Performance of the approximation

\begin{tabular}{lllllll}
\hline Stage 1 & & $\pi^{s}$ & $\begin{array}{l}\text { Expected } \\
\text { subcon- } \\
\text { tracted }\end{array}$ & $\begin{array}{l}\text { Expected end- } \\
\text { ing on-hand } \\
\text { inv. }\end{array}$ & $\begin{array}{l}\text { Expected WIP } \\
\text { Inv. }\end{array}$ & Expected backlog \\
\hline $\mathrm{p}_{1}=0.05$ & Exact & 0.9975 & 0.0001 & 0.9476 & 0.0525 & 0.0026 \\
$\mathrm{p}_{2}=0.05$ & Approximation & 0.9975 & 0.0001 & 0.9478 & 0.0525 & 0.0026 \\
$\mathrm{p}_{1}=0.1$ & Exact & 0.9902 & 0.0011 & 0.8910 & 0.1099 & 0.0109 \\
$\mathrm{p}_{2}=0.1$ & Approximation & 0.9903 & 0.0011 & 0.8920 & 0.1099 & 0.0108 \\
$\mathrm{p}_{1}=0.1$ & Exact & 0.9908 & 0.0010 & 0.8977 & 0.1100 & 0.0102 \\
$\mathrm{p}_{2}=0.05$ & Approximation & 0.9908 & 0.0010 & 0.8979 & 0.1100 & 0.0102 \\
$\mathrm{p}_{1}=0.1$ & Exact & 0.9878 & 0.0014 & 0.8640 & 0.1096 & 0.0136 \\
$\mathrm{p}_{2}=0.2$ & Approximation & 0.9885 & 0.0014 & 0.8716 & 0.1096 & 0.0128 \\
$\mathrm{p}_{1}=0.2$ & Exact & 0.9629 & 0.0093 & 0.7680 & 0.2384 & 0.0464 \\
$\mathrm{p}_{2}=0.2$ & Approximation & 0.9642 & 0.0091 & 0.7755 & 0.2386 & 0.0449 \\
$\mathrm{p}_{1}=0.05$ & Exact & 0.9958 & 0.0002 & 0.9120 & 0.0524 & 0.0044 \\
$\mathrm{p}_{2}=0.2$ & Approximation & 0.9962 & 0.0002 & 0.9197 & 0.0524 & 0.0040 \\
$\mathrm{p}_{1}=0.05$ & Exact & 0.9972 & 0.0001 & 0.9405 & 0.0525 & 0.0030 \\
$\mathrm{p}_{2}=0.1$ & Approximation & 0.9972 & 0.0001 & 0.9415 & 0.0525 & 0.0029 \\
$\mathrm{p}_{1}=0.2$ & Exact & 0.9667 & 0.0083 & 0.7920 & 0.2396 & 0.0416 \\
$\mathrm{p}_{2}=0.1$ & Approximation & 0.9669 & 0.0083 & 0.7931 & 0.2396 & 0.0414 \\
$\mathrm{p}_{1}=0.2$ & Exact & 0.9677 & 0.0081 & 0.7980 & 0.2399 & 0.0404 \\
$\mathrm{p}_{2}=0.05$ & Approximation & 0.9677 & 0.0081 & 0.7982 & 0.2399 & 0.0404 \\
\hline
\end{tabular}

\subsection{Computational results via enumeration}

In Sect. 4.2 we present an algorithm to compute steady-state probabilities for each stage given $K$ and $M$ values for different stages. We restrict our computations to two-stage systems only. We approach the problem by stating a service level for Stage 1 . Note that we enumerate all possible decisions (in this case $K$ 's, and $M$ 's if a stage is GSM) that will yield the desired service level for Stage 1. Here, the service level the end customer faces corresponds to $\pi^{S_{1}}$ by definition.

Before enumerating all possible solutions, one can compare the quantities of the GSM regime with those of the SSM regime given a fixed supply probability. The following observations are made: As $M$ increases, expected finished goods inventory decreases for the GSM since probabilities for states with less finished goods inventory are larger, whereas expected work-in-process inventory increases. As $K$ increases, finished goods inventory increases for both regimes. However, as $K$ varies, expected work-in-process inventory stays the same for both regimes, as work-in-process is related to supply probability of the upstream stage. The SSM regime keeps less finished goods inventory and more work-in-process inventory than the GSM regime for the same $\pi^{S_{1}}$ value whereas the supply probability of GSM is greater than the supply probability of the corresponding SSM. Finally, the SSM has more expected backlogs than the corresponding GSM. 
In the numerical analyses we consider combinations of various SSM and GSM systems for both stages and compute the expected total cost of inventories, and effects on the expected outsourcing quantities.

Expected total ending on-hand inventory cost is found according to either Eqs. (9) or (13) for Stage 1 and according to either Eqs. (2) or (4) for Stage 2. We set inventory holding cost as 1 for Stage 1 and 0.5 for Stage 2. Hence, expected ending on-hand inventory cost is found by multiplying holding costs by ending on-hand inventory amounts. For Stage 1, expected work-in-process inventory is again found according to Eqs. (10) or (14) and is included in the expected total ending on-hand inventory cost. Expected backlog quantities are calculated according to Eqs. (11) or (16).

The values considered for numerical examples are as follows: For any stage and any regime $K=1,2$, and for the GSM regime at any stage $M=K, K+1, K+2, K+3$. In total 100 combinations of these 10 different cases are evaluated when the probability of no production is taken as 0.2 for all cases.

\subsubsection{Expected total carrying cost}

For ease of presentation, we clustered the comparison cases according to the service level achieved by Stage 1. The service level ranges from $95.3 \%$ to $96.8 \%$ and from $99 \%$ to $100 \%$. Therefore, we clustered the cases according to the minimum service level obtained (95.3\% and 99\%). Detailed results for the best 10 combinations based on "expected total ending on-hand inventory costs" are presented in Tables 6 and 7 in "Appendix 8", following other work in the area that presents only these costs (Graves and Willems 2003). As expected, SSM regime yields lower expected on-hand inventory cost values when the service level is not high. When it is high, we expect the GSM to be relatively effective.

Service level is [95.3\%, 96.8\%] Table 6 in "Appendix 8" ranks the models when the service level of the system is around $95.3 \%$. When both stages are operated under the SSM and $K_{1}=K_{2}=1$, the system has the minimum expected total ending on-hand inventory cost. It can be observed that the GSM when $K_{2}=1$ for Stage 2 performs better than other combinations. The best combination for the GSM is when $K_{2}=1$ and $M_{2}=4$ (the limit we tried, and hence closest to the SSM regime).

Service level is [99 \%, 100\%] Table 7 in "Appendix 8" ranks the cases in increasing order of the total expected ending on-hand inventory cost where the service level of the system is around $99 \%$. The best case is an SSM/GSM combination when $K_{2}=1$ and $K_{1}=1, M_{1}=1$. The explanation is straightforward: As the SSM keeps more stock for the required service level, a combination that uses the GSM regime outperforms an SSM/SSM system.

\subsubsection{Measuring effects of outsourcing}

One of the assumptions made by Graves and Willems (2003) for the GSM regime, is that the excess demand over the accepted maximum bound is outsourced from another source to satisfy customer requirements. However, there is no additional term that represents the outsourced amount in the objective function, assuming that 
the expected subcontracting cost will be negligible. Note that we followed the same assumption in our work. Here we analyze the effect of that assumption.

For the analysis, we consider two systems where one system uses the GSM regime only (call it the GSM/GSM system) and the other one uses the SSM regime (call it the SSM/SSM system). Note that in order to make a reasonable comparison, the expected ending on-hand inventory cost of the SSM/SSM system should be larger than that of the GSM/GSM system, while both should yield approximately the same service level. Given these conditions, our aim is to find a threshold outsourcing cost per unit for Stage 1 (the outsourcing cost for Stage 2 has the same proportion as holding costs, i.e., $=h_{2} / h_{1}$ ), which will yield the same expected costs for both systems.

To come up with a comparison, we consider minimum service level clusters $95.3 \%$ and $99 \%$, and the SSM/SSM system with $K_{2}=2, K_{1}=1$ and $K_{2}=2, K_{1}=2$, respectively. Those are the cases that yield a higher expected on-hand inventory cost compared to some GSM/GSM combinations. We take the difference of expected ending on-hand inventory costs and divide the difference by the cost of total net expected outsourced in the GSM/GSM system (expected outsourced in Stage $1+$ $\left(h_{2} / h_{1}\right)^{*}$ expected outsourced in Stage 2$)$ to find the threshold outsourcing cost that will equate the expected total cost of the two cases considered. Using the threshold cost found, we compute the expected cost of subcontracting as a percentage of total expected cost of ending on-hand inventory, and we represent the threshold cost as a percentage of the expected backlog cost implied by the service level used for the stage. The results are shown in Tables 4 and 5 .

Table 4 Analyses with threshold outsourcing costs when service level is at least 95.3\% (range: [95.3\%, $96.8 \%])$

\begin{tabular}{lllllll}
\hline $\begin{array}{l}\text { Case } \\
\text { number }\end{array}$ & $K_{2}$ & $M_{2}$ & $K_{1}$ & $M_{1}$ & $\begin{array}{l}\text { Total expected subcontracting } \\
\text { cost at threshold as a percentage } \\
\text { of total expected ending on-hand } \\
\text { inventory holding cost }(\%)\end{array}$ & $\begin{array}{l}\text { Total expected subcontract- } \\
\text { ing cost at threshold as a } \\
\text { percentage of unit total } \\
\text { expected backlog cost }(\%)\end{array}$ \\
\hline 1 & 1 & 4 & 2 & 4 & 15 & 28 \\
2 & 1 & 4 & 2 & 5 & 7 & 6 \\
3 & 1 & 3 & 2 & 4 & 7 & 13 \\
Base case & 2 & NA & 2 & NA & - & - \\
\hline
\end{tabular}

Table 5 Analyses with threshold outsourcing costs when service level is at least 99\% (range: [99\%, $100 \%]$ )

\begin{tabular}{lllllll}
\hline Case number & $K_{2}$ & $M_{2}$ & $K_{1}$ & $M_{1}$ & $\begin{array}{l}\text { Total expected subcontract- } \\
\text { ing cost at threshold as a } \\
\text { percentage of total expected } \\
\text { ending on-hand inventory } \\
\text { holding cost }(\%)\end{array}$ & $\begin{array}{l}\text { Total expected subcon- } \\
\text { tracting cost at threshold } \\
\text { as a percentage of unit } \\
\text { total expected backlog } \\
\text { cost (\%) }\end{array}$ \\
\hline 1 & 1 & 2 & 1 & 2 & 23 & 26 \\
2 & 1 & 3 & 1 & 2 & 30 & 35 \\
3 & 1 & 4 & 1 & 2 & 32 & 38 \\
Base case & 2 & NA & 1 & NA & - & - \\
\hline
\end{tabular}


Both results indicate that it is beneficial to take into account the effect of outsourced quantity in certain cases, as opposed to not considering it at all. Especially when higher service levels are considered, subcontracting is beneficial with a relatively low threshold value, which implies that the GSM regime is likely to be a more expensive choice.

\section{Conclusions and future work}

For the single-stage system, we can evaluate steady-state probabilities of a DTMC exactly when service regime parameters are ( $K$ for the SSM and $K$ and $M$ for the GSM) supplied. The two-stage system can also be represented exactly by a DTMC, when each stage is allowed to follow either the SSM or the GSM regime. However, when it comes to evaluating steady-state probabilities, the number of states grow exponentially prohibiting sound computations. Nevertheless, we formulate a GSM/ GSM operated two-stage system and compute the steady-state probabilities exactly. Once we have steady-state probabilities, we can obtain quantities for expected ending on-hand inventory of finished goods and work-in-process inventories, as well as expected backlogs and expected outsourced amount.

For more realistic serial systems, we offer an approximation. We decompose the stages by defining the probability of supply from one stage to the next. We numerically show that the decomposition approximation is fairly accurate for the two-stage model.

To analyze the systems further, we use total enumeration considering all combinations (GSM, SSM for any stage) of service regimes with varying $K$ and $M$ values. We cluster combinations that yield reasonably close service levels and compare different systems. Results suggest that for reasonable service levels, an SSM/SSM system results in the lowest total expected on-hand inventory costs. However, when the service level is relatively higher, we expect the GSM to be relatively effective.

Finally, we make some computations to test the assumption made in the literature that expected subcontracting cost is negligible. We conclude by showing that expected quantity to outsource may well be an important consideration, and hence should not be ignored.

One immediate extension of the current work is to model a two echelon assembly system. As all suppliers operate according to a base stock policy and a service model (SSM or GSM), the probability of supply for each supplier can be obtained by solving the single-stage problem, as in the two-stage serial system. Therefore, the system can be modeled by using a DTMC structure and probability values can be used in the transitions to reflect a supplier's effect on Stage 1. The difference between a serial system and an assembly system is in the state representation of Stage 1: Each supplier's work-in-process inventory in stock should be represented. The size of the state space grows rapidly in this case, and hence solving this problem even computationally may require a different method. Decomposing the model [see de Kok and Visschers (1999)] appears to be an acceptable direction to proceed, although a modification method used in Ettl et al. (2000) can be pursued as well. 
Future work can also consider the partial supply (production) availability case. Most features of the model may remain the same, except the reflection of the supply uncertainty structure. In the current context, supply uncertainty that the downstream stage faces is a result of production uncertainty at the upstream stage. Hence, supply unavailability can be used to reflect production unavailability. In the extension, desired production quantity may be fully supplied, partially supplied or not supplied at all. Following the structure of Güllü et al. (1999) a DTMC can be specified requiring an extension to the already existing states.

\section{Appendix 1: Probability transition matrix for single-stage problem under the SSM}

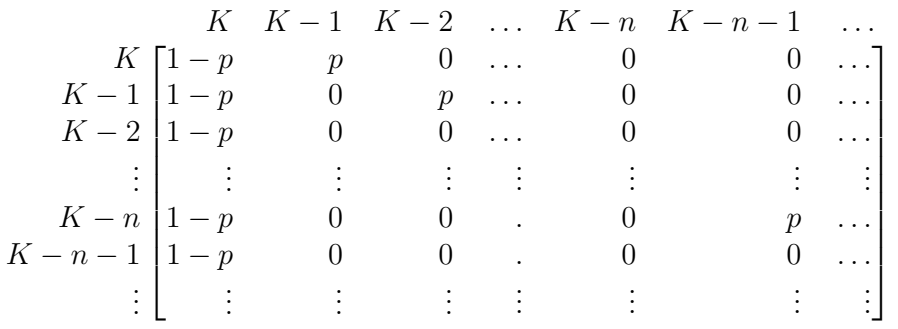

\section{Appendix 2: Probability transition matrix for single-stage problem under the GSM}

\begin{tabular}{|c|c|c|c|c|c|c|c|}
\hline & $K$ & $K-1$ & $K-2$ & $K-3$ & $\ldots$ & $-M+K+1$ & $-M+K$ \\
\hline$K$ & $\lceil 1-p$ & $p$ & 0 & 0 & $\ldots$ & 0 & 07 \\
\hline$K-1$ & $1-p$ & 0 & $p$ & 0 & $\ldots$ & 0 & 0 \\
\hline$K-2$ & $1-p$ & 0 & 0 & $p$ & $\ldots$ & 0 & 0 \\
\hline & . & $\vdots$ & . & $\vdots$ & $\vdots$ & & \\
\hline$M+K+1$ & $1-p$ & 0 & 0 & 0 & $\ldots$ & 0 & $p$ \\
\hline$-M+K$ & $\lfloor 1-p$ & 0 & 0 & 0 & $\ldots$ & 0 & $p$ \\
\hline
\end{tabular}




\section{Appendix 3: Probability transition matrix for two-stage problem} under the GSM

$\left.\begin{array}{rrrrrrrrr}(1,1,0) & (1,0,1) & (1,-1,2) & (0,0,1) & (0,1,0) & (0,-1,2) & (-1,0,0) & (-1,-1,1) \\ (1,1,0) & \left(1-p_{1}\right)\left(1-p_{2}\right) & \left(1-p_{2}\right) p_{1} & 0 & p_{1} p_{2} & \left(1-p_{1}\right) p_{2} & 0 & 0 & 0 \\ (1,0,1) \\ (1,-1,2) \\ (0,0,1) \\ \left(1-p_{1}\right)\left(1-p_{2}\right) & 0 & \left(1-p_{2}\right) p_{1} & 0 & \left(1-p_{1}\right) p_{2} & p_{1} p_{2} & 0 & 0 \\ (0,1,0) & \left(1-p_{1}\right)\left(1-p_{2}\right) & 0 & \left(1-p_{2}\right) p_{1} & 0 & \left(1-p_{1}\right) p_{2} & p_{1} p_{2} & 0 & 0 \\ (0,-1,2) \\ \left(1-p_{1}\right)\left(1-p_{2}\right) & 0 & \left(1-p_{2}\right) p_{1} & 0 & 0 & 0 & \left(1-p_{1}\right) p_{2} & p_{1} p_{2} \\ (-1,0,0) & \left(1-p_{1}\right)\left(1-p_{2}\right) & \left(1-p_{2}\right) p_{1} & 0 & 0 & 0 & 0 & p_{2} & 0 \\ (-1,-1,1) & \left(1-p_{1}\right)\left(1-p_{2}\right) & 0 & \left(1-p_{2}\right) p_{1} & 0 & 0 & 0 & \left(1-p_{1}\right) p_{2} & p_{1} p_{2} \\ \left(1-p_{1}\right)\left(1-p_{2}\right) & 0 & \left(1-p_{2}\right) p_{1} & 0 & 0 & 0 & \left(1-p_{1}\right) p_{2} & p_{1} p_{2} \\ \left(1-p_{1}\right)\left(1-p_{2}\right) & 0 & \left(1-p_{2}\right) p_{1} & 0 & 0 & 0 & \left(1-p_{1}\right) p_{2} & p_{1} p_{2}\end{array}\right]$

Appendix 4: Two-stage model: expressions for stage 1 for the SSM

\section{SSM}

Replenishment Time $(\tau)$ $\tau=\left\{i\right.$ with probability $\left.\pi^{S_{2}}\left(1-p_{1}\right)\left(\sum_{j=0}^{i}\left[\left(1-\pi^{S_{2}}\right)^{i-j} p_{1}^{j}\right]\right)\right\}$

Demand during $\tau$ where $\mathrm{i}=0,1,2,3, \ldots$ $D=\left\{i Q\right.$ with probability $\left.\pi^{S_{2}}\left(1-p_{1}\right)\left(\sum_{j=0}^{i}\left[\left(1-\pi^{S_{2}}\right)^{i-j} p_{1}^{j}\right]\right)\right\}$
$E\left[D_{\tau}\right]$
$Q \pi^{S_{2}}\left(1-p_{1}\right)\left(\sum_{i=1}^{\infty} \sum_{j=0}^{i}\left[i\left(1-\pi^{S_{2}}\right)^{i-j} p_{1}^{j}\right]\right)$
$\sigma_{\tau}^{2}$
$Q^{2} \pi^{S_{2}}\left(1-p_{1}\right)\left(\sum_{i=1}^{\infty} \sum_{j=0}^{i}\left[i^{2}\left(1-\pi^{S_{2}}\right)^{i-j} p_{1}^{j}\right]\right)$
$-\left[Q \pi^{S_{2}}\left(1-p_{1}\right)\left(\sum_{i=1}^{\infty} \sum_{j=0}^{i}\left[i\left(1-\pi^{S_{2}}\right)^{i-j} p_{1}^{j}\right]\right)\right]^{2}$ 


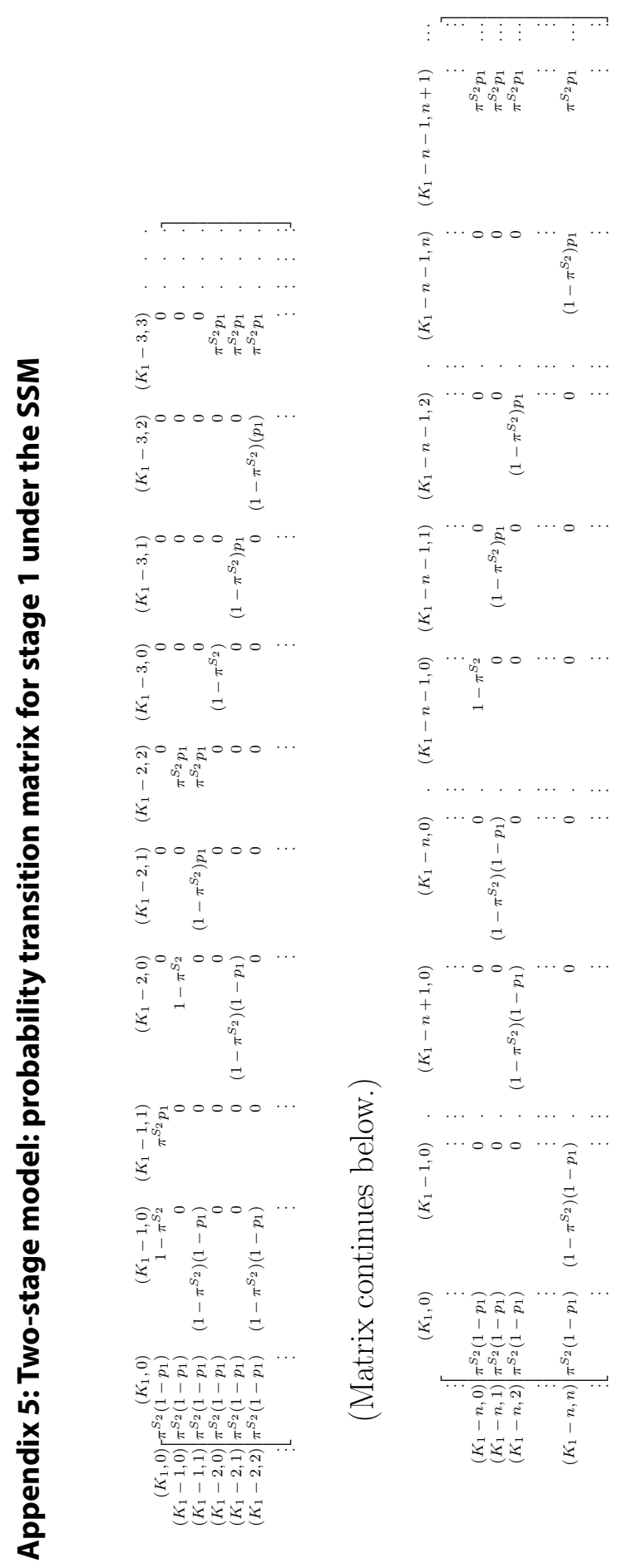




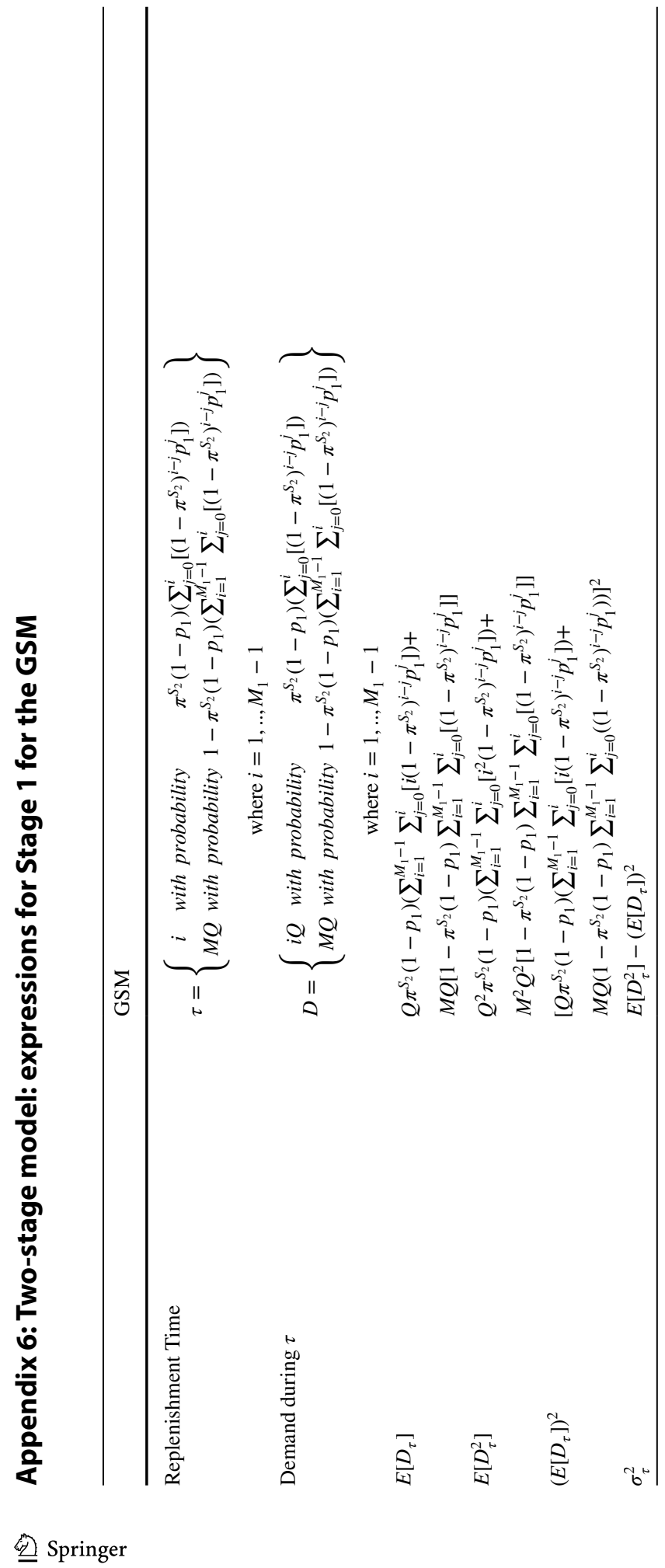




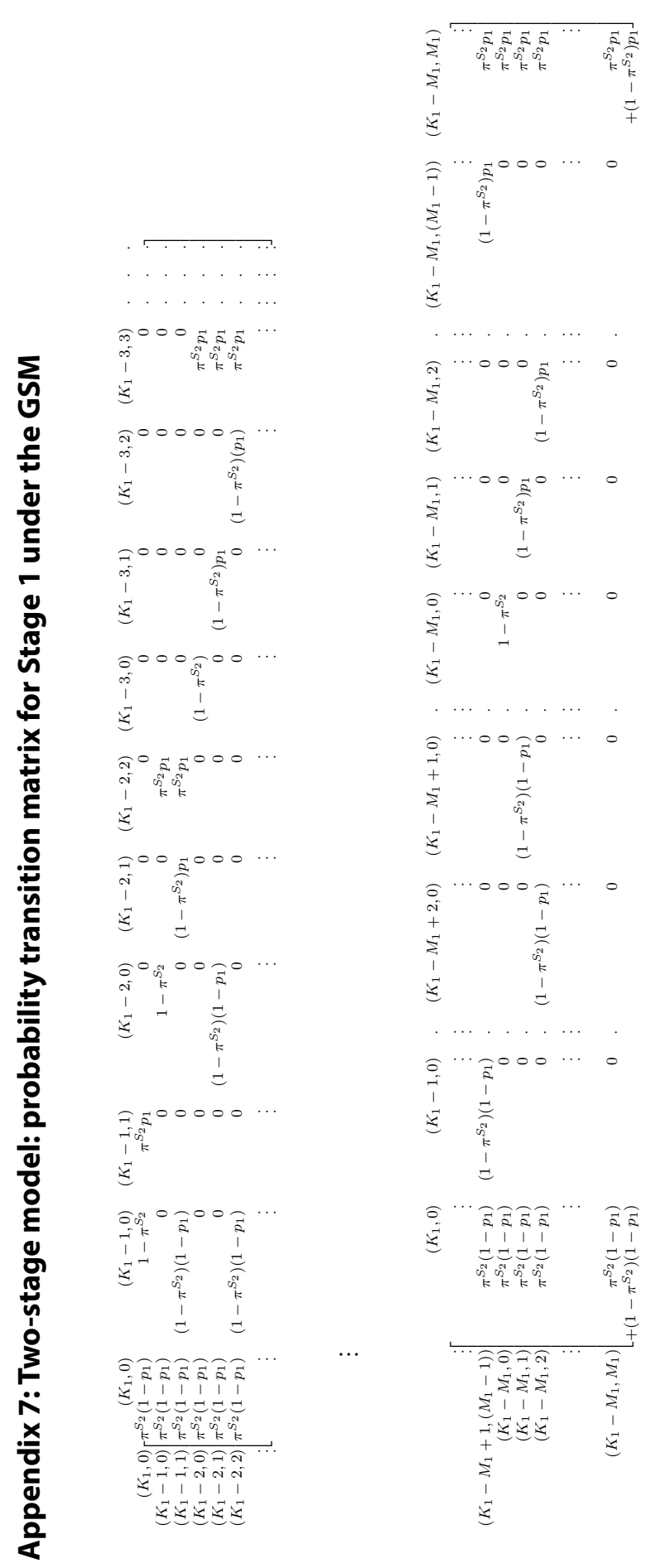




\section{Appendix 8: Two-stage models: ranking according to expected total on-hand inventory costs}

See Tables 6 and 7.

Table 6 Service level is at least $95.3 \%$ (range: $[95.3 \%, 96.8 \%])$

\begin{tabular}{lllllll}
\hline $\begin{array}{l}\text { Total expected ending } \\
\text { on-hand inventory } \\
\text { cost }\end{array}$ & $\begin{array}{l}\text { Stage 2 } \\
\text { service } \\
\text { model }\end{array}$ & $\begin{array}{l}\text { Stage } 1 \\
\text { service } \\
\text { model }\end{array}$ & $\mathrm{K}_{2}$ & $\mathrm{M}_{2}$ & $\mathrm{~K}_{1}$ & $\mathrm{M}_{1}$ \\
\hline 1 (min) & SSM & SSM & 1 & NA & 1 & NA \\
2 & GSM & SSM & 1 & 4 & 1 & NA \\
3 & GSM & SSM & 1 & 3 & 1 & NA \\
4 & GSM & SSM & 1 & 2 & 1 & NA \\
5 & GSM & SSM & 1 & 1 & 1 & NA \\
6 & SSM & GSM & 1 & NA & 1 & 2 \\
7 & GSM & GSM & 1 & 4 & 1 & 2 \\
8 & GSM & GSM & 1 & 3 & 1 & 2 \\
9 & GSM & GSM & 1 & 2 & 1 & 2 \\
10 & SSM & GSM & 1 & NA & 1 & 3 \\
\hline & & & & & &
\end{tabular}

Table 7 Service level is at least 99\% (range: [99\%, 100\%])

\begin{tabular}{lllllll}
\hline $\begin{array}{l}\text { Total expected ending on- } \\
\text { hand inventory cost }\end{array}$ & $\begin{array}{l}\text { Stage 2 service } \\
\text { model }\end{array}$ & $\begin{array}{l}\text { Stage 1 service } \\
\text { model }\end{array}$ & $\mathrm{K}_{2}$ & $\mathrm{M}_{2}$ & $\mathrm{~K}_{1}$ & $\mathrm{M}_{1}$ \\
\hline $1(\mathrm{~min})$ & SSM & GSM & 1 & NA & 1 & 1 \\
2 & GSM & GSM & 1 & 4 & 1 & 1 \\
3 & GSM & GSM & 1 & 3 & 1 & 1 \\
4 & GSM & GSM & 1 & 2 & 1 & 1 \\
5 & GSM & GSM & 1 & 1 & 1 & 1 \\
6 & SSM & GSM & 2 & NA & 1 & 1 \\
7 & GSM & GSM & 2 & 5 & 1 & 1 \\
8 & GSM & GSM & 2 & 4 & 1 & 1 \\
9 & GSM & GSM & 2 & 3 & 1 & 1 \\
10 & GSM & GSM & 2 & 2 & 1 & 1 \\
\hline
\end{tabular}

\section{References}

Atan Z, Ahmadi T, Stegehuis C, de Kok T, Adan I (2017) Assemble-to-order systems: a review. Eur J Oper Res 261(3):866-879

Axsäter S (2003) Supply chain operations: serial and distribution inventory systems. Handb Oper Res Manag Sci 11:525-559

Bollapragada R, Rao US, Zhang J (2004a) Managing inventory and supply performance in assembly systems with random supply capacity and demand. Manag Sci 50(12):1729-1743

Bollapragada R, Rao US, Zhang J (2004b) Managing two-stage serial inventory systems under demand and supply uncertainty and customer service level requirements. IIE Trans 36(1):73-85

de Kok TG, Visschers J (1999) Analysis of assembly systems with service level constraints. Int J Prod Econ 59(1-3):313-326 
Diamantidis A, Papadopoulos C (2004) A dynamic programming algorithm for the buffer allocation problem in homogeneous asymptotically reliable serial production lines. Math Probl Eng 2004(3):209-223

Ehrhardt R, Taube L (1987) An inventory model with random replenishment quantities. Int J Prod Res 25(12):1795-1803

Ettl M, Feigin GE, Lin GY, Yao DD (2000) A supply network model with base-stock control and service requirements. Oper Res 48(2):216-232

Gavirneni S (2004) Supply chain management at a chip tester manufacturer. In: Harrison TP, Lee HL, Neale JJ (eds) The practice of supply chain management: where theory and application converge. Springer, Berlin, pp 277-291

Gershwin SB (1987) An efficient decomposition method for the approximate evaluation of tandem queues with finite storage space and blocking. Oper Res 35(2):291-305

Gershwin SB, Schor JE (2000) Efficient algorithms for buffer space allocation. Ann Oper Res 93(1-4):117-144

Graves SC, Willems SP (2003) Supply chain design: safety stock placement and supply chain configuration. Handb Oper Res Manag Sci 11:95-132

Graves SC, Willems SP (2005) Optimizing the supply chain configuration for new products. Manag Sci 51(8):1165-1180

Güllü R, Önol E, Erkip N (1997) Analysis of a deterministic demand production/inventory system under nonstationary supply uncertainty. IIE Trans 29(8):703-709

Güllü R, Önol E, Erkip N (1999) Analysis of an inventory system under supply uncertainty. Int J Prod Econ 59(1-3):377-385

Henig M, Gerchak Y (1990) The structure of periodic review policies in the presence of random yield. Oper Res 38(4):634-643

Hua NG, Willems SP (2016a) Analytical insights into two-stage serial line supply chain safety stock. Int J Prod Econ 181:107-112

Hua NG, Willems SP (2016b) Optimally configuring a two-stage serial line supply chain under the guaranteed service model. Int J Prod Econ 181:98-106

Inderfurth K, Minner S (1998) Safety stocks in multi-stage inventory systems under different service measures. Eur J Oper Res 106(1):57-73

Lee JH, Li J, Horst JA (2017) Serial production lines with waiting time limits: Bernoulli reliability model. IEEE Trans Eng Manag 65(2):316-329

Lee JH, Zhao C, Li J, Papadopoulos CT (2018) Analysis, design, and control of Bernoulli production lines with waiting time constraints. J Manuf Syst 46:208-220

Liberopoulos G (2018) Performance evaluation of a production line operated under an echelon buffer policy. IISE Trans 50(3):161-177

Naebulharam R, Zhang L (2014) Bernoulli serial lines with deteriorating product quality: performance evaluation and system-theoretic properties. Int J Prod Res 52(5):1479-1494

Parlar M, Berkin D (1991) Future supply uncertainty in EOQ models. Naval Res Logist (NRL) 38(1): 107-121

Rambau J, Schade K (2014) The stochastic guaranteed service model with recourse for multi-echelon warehouse management. Math Methods Oper Res 79(3):293-326

Shih W (1980) Optimal inventory policies when stockouts result from defective products. Int J Prod Res 18(6):677-686

Silver E (1976) Establishing the order quantity when the amount received is uncertain. INFOR: Inf Syst Oper Res 14(1):32-39

Snyder LV, Atan Z, Peng P, Rong Y, Schmitt AJ, Sinsoysal B (2016) Or/ms models for supply chain disruptions: a review. IIE Trans 48(2):89-109

Song JS, Zipkin P (2003) Supply chain operations: assemble-to-order systems. Handb Oper Res Manag Sci 11:561-596

Weiss S, Schwarz JA, Stolletz R (2019) The buffer allocation problem in production lines: formulations, solution methods, and instances. IISE Trans 51(5):456-485

Wu K, Shen Y, Zhao N (2017) Analysis of tandem queues with finite buffer capacity. IISE Trans 49(11):1001-1013

Yano CA, Lee HL (1995) Lot sizing with random yields: a review. Oper Res 43(2):311-334

Publisher's Note Springer Nature remains neutral with regard to jurisdictional claims in published maps and institutional affiliations. 
Bengisu Urlu completed M.S. in Operations Management and Logistics at Eindhoven University of Technology, and B.S in Industrial Engineering at Bilkent University. She is currently a Ph.D. student in Technology and Operations Management area at INSEAD. Her research interests include supply chain operations, especially in the context of humanitarian and sustainable supply chains.

Nesim K. Erkip received M.S. and Ph.D. from Stanford University and B.S. from Middle East Technical University (METU), Ankara. Prior to joining Bilkent University in 2005, he worked at the METU for over 20 years. He held visiting and research positions at Cornell University, Stanford University, UC Berkeley, Eindhoven University of Technology, New York University and Technical University of Munich. His main research interests are multi-echelon inventory theory, distribution systems, supply chains and retailing, as well as applications of OR. 